"Professional" Acts: Analyzing sites of identity and interactive response in chemical engineering students

Ms. Deborah Tihanyi, University of Toronto

Deborah Tihanyi is a Senior Lecturer in the Engineering Communication Program.

Dr. Penny Kinnear, University of Toronto 


\section{"Professional" Acts: Analyzing sites of identity and interactive response in chemical engineering students}

In September 2012, we began a five-year longitudinal study following eleven (11) Chemical Engineering and Applied Chemistry (CHE) students who began their second year of study in 2012-2013. The study focuses on the development of professional identity and voice in CHE students at the University of Toronto. In this paper, we will present an analysis of one participant's experience in two related sites of learning, identity and interactive response, which will allow us to showcase early "professional" acts, the responses to them, and the ways in which they evolved over the first year of the study period.

It is important to note that we have only been able to look closely at a small portion of the data collected in the first year of this five-year, longitudinal study. As a result, we are not yet in a position to extrapolate, responsibly draw firm conclusions or identify trends, nor can we identify specific curricular or pedagogical implications. What we can do at this stage is highlight some of our initial findings that will inform the analysis of the rest of the data. In this paper, we focus on Téa, ${ }^{1}$ one of eleven participants, based on the artifacts collected to date, although reference will be made to comments and work of other participants. We hope to show through this preliminary analysis how one student uses the experiences and opportunities provided both by the curriculum and this research project to develop a sense of professionalism and how to practice it as a chemical engineer as she tries on/tries out versions of her chemical engineer identity.

\section{Background}

The motivation for the study goes back several years, as a result of our involvement with the CHE portfolio courses (CHE297 and CHE397). These courses afforded an opportunity for students to synthesize their learning in core courses, consider the relationships between what they were studying and their future careers and develop their written and oral communication skills. What emerged from that experience was the sense that there were significant changes happening in terms of how students viewed themselves as students and potential engineers; what we wanted to discover was what was happening in the curriculum - and what was, perhaps, inherent in the chemical engineering culture - to facilitate those changes. Through targeted focus groups with current and former students and examination of student portfolios from previous years, we identified a number of key areas, including

- the nature of the chemical engineering culture/community, especially at the University of Toronto,

- the practices that define chemical engineering and engineers, and

- the tensions that exist between academic requirements, industry expectations and students' sense of their educational needs.

What we found in that initial research was a close-knit community of students, faculty and staff, distinguished within the Faculty of Applied Science and Engineering (FASE) by their emphasis on professional skills (especially communication and leadership) in addition to the technical and scientific foundations of the discipline.

${ }^{1}$ A pseudonym, as are all participant names—-students and faculty—used throughout this paper. 
Professional identity (and its development) is a current topic in international engineering education research, although there is not a comprehensive body of work to date. Karen L. Tonso's ethnographic study ${ }^{[1]}$ included her participation in engineering student teamwork over three years. Her findings highlight the complexity of the notion of "engineering identity," involving the interplay of perceptions of engineering culture (inside and outside the university program), the practice of engineering activity and the measuring of self among these multiple contexts. Given this complexity, as Eisenhart and Brickhouse ${ }^{[2]}$ suggest in their commentary on Tonso's study, it is necessary to broaden the scope of our research beyond discrete situations, indeed to include multiple sites of learning over time.

Also serving to make the study timely is the Canadian Engineering Accreditation Board's (CEAB) move to outcomes based assessment and FASE's concomitant review of graduate attributes serving to develop curriculum to support these outcomes (of particular interest to this study are communication skills, professionalism and life-long learning). Our key objectives are to:

- Examine how chemical engineering identities are developed, expressed, conceptualized and instantiated.

- Provide insight into how students and instructors experience and make use of the elements of the curriculum in Chemical Engineering and Applied Chemistry in the recognition and development of the students' professional identity and voice.

- Inform curriculum design to support the development of professional identity in students across the Faculty.

\section{Identity}

We entered into this study using the assumptions that identity is understood as a process in which individuals construct, reconstruct and develop multiple identities in cooperation with or in resistance to the contexts in which the individuals place themselves or are placed ${ }^{[3]}$. In 1986 Markus and Nurius ${ }^{[4]}$ referenced a considerable body of work that conceptualized identity and self-concept as complex and dynamic but noted that the empirical work to support those conceptualizations remained mostly undone. That left the traditional view of self-concept as "[...] a fairly uniform, monolithic structure, consistent over time, comprising some number of physical features of psychological structures that abstract the essential traits from the individual's past behavior" ${ }^{[4]}$ as the dominant conceptualization. Part of the complexity of identity stems from the now generally accepted idea that an individual can have multiple identities, a portfolio or repertoire of identities, as it were. However, much of the empirical work that has been done with regard to the more complex and dynamic conceptualization of identity has occurred within organizations and focused on the context of someone already in a career or a profession adapting to or complying with new roles and responsibility requirements (see Ibarra, 1999 ${ }^{[5]}$ and Ibarra and Petriglieri $2010^{[6]}$ for a more complete review of this). Research focused on students before they have committed to a particular professional identity has been sparse although there are recent exceptions ${ }^{[1,6]}$. Much of this work has focused on women in engineering or the retention of students in the field. Ibarra and Petriglieri make two additional relevant points about this identity research. First it has focused on "central, actual and present identities" ${ }^{p]}$ and second, on how people "create coherence and continuity across their various identities"[6]. The undergraduate years present a kind of liminal space (Turner, 1969 as cited in Ibarra and Petriglier ${ }^{[6]}$ ). This is 
because students are more likely to be in a position where they are considering or exploring future identities, who or what they will or want to become.

According to Eliot and Turns ${ }^{[7]}$, although professional identity development is considered to be essential for engineering students, it is not an explicit goal in engineering curricula but rather more of, in their words, an "epiphenomenon"[7]. As such what students do to form these professional identities has not been a major subject of investigation. Our analysis of our data around the development of professional identity is grounded in the dynamic conceptualization of identity development rather than the more traditional, static one. In particular, we draw upon a combination of Markus and Nurius and Ibarra's work that deals with the idea of possible selves and identity play to examine this process.

\section{Interactive Response and Interactions}

From our early observations of the students' use of the portfolios, we could see that identity work and play occur in a variety of contexts, mediated by individual reflections as well as the responses and interactions of other individuals. These observations informed our categorization of "Interactive Response" (IR) as a site of learning mediated by diverse interactions with both people (instructors, peers, friends/family, clients) and symbolic artifacts (e.g., professional codes of ethics). In this conception of IR, we pick up on Hattie and Timperley's broader notion of feedback as provided by multiple "agent $[\mathrm{s}] "[8]$ in response to a particular student act.

Discussion of feedback and assessment in recent years has placed a great deal of emphasis on the consideration of student needs at particular times in the learning process ${ }^{[9]}$. This diversity of needs can be addressed through the use of multiple tools of assessment, a focus on practice and the opportunity for students to reflect on their learning ${ }^{[9]}$. As Hattie points out, "the most powerful single moderator that enhances achievement is feedback" ${ }^{[10]}$. However, he distinguishes between two elements of instructor-student interaction: feedback, which is material related to "the constructions th[e] students have made from the information",[10] and reinforcement, which is "the evaluative component relating to information and motivation"[10]. The presence of these two elements, both the informative/formative discussions that lead to development and the evaluative/summative commentary that assesses a student's standing, is necessary in order for learning to take place ${ }^{[10,11]}$.

We understand feedback and assessment primarily from a sociocultural perspective that places emphasis on the mediational nature of the kinds of feedback activities Hattie describes. We place the feedback activities in a broader category of potential scaffolding activities as first defined by Wood, Bruner and Ross in $1976^{[12]}$. These activities or more accurately, interactions, provide opportunities for learners to imitate, to participate, to practice, and to develop understandings. Although the literature on the uptake of feedback, both oral and written, on student work is inconclusive ${ }^{[13,14,15]}$, what is increasingly clear is the role of contingent interaction now often referred to as dynamic assessment or dynamic teaching ${ }^{[16,17,18,19]}$ in enabling students to become independent in their mastery of knowledge and skills as well as the learning process itself.

We deliberately chose the term "Interactive Response" to highlight the complex nature of "feedback," one that encompasses the diversity of student needs and the nature of the 
interactions between students and their interlocutors in the learning process. Most importantly, through an analysis of our data, we have been able to see how the sites of identity and interactive response overlap.

\section{Methodology}

We are following a cohort of 11 (eleven) chemical engineering students. We began in September 2012, as the students entered their second year of engineering (and their first year in the discipline of chemical engineering) and we will continue to follow them until one year after graduation. The students form the core of the participants. Additional participants include the faculty, professors and teaching assistants, involved in selected courses. Courses included in the study were chosen based on preliminary research (interviews and document analysis) that identified certain courses as pivotal to the transition from student to professional. Data include individual interviews, discussion groups, written and oral, individual and team assignments, rubrics, feedback, language logs and participant observation. (For a more detailed account, see Appendix A.)

\section{Preliminary Findings}

Most students do not enter the engineering program with a well-developed sense of what an engineer does - the day-to-day tasks, the responsibilities the work entails or the social and legal responsibilities incumbent upon professional engineers. Many enter the program because they are good at math and physics or because a family member is an engineer or simply because it is a profession and the student (and the student's family) assume that the student will immediately enter into a well-paying and secure position. We understand the undergraduate years as a liminal space or time ${ }^{[4,7]}$ during which students can explore possible selves and possible professional identities. Ibarra and Petriglieri characterize this kind of activity as identity play, a characterization we share. They define identity play as "people's engagement in provisional but active trial of possible future selves" ${ }^{,[6]}$. We have identified a number of course experiences as potential sites for this identity play. These include:

- the lab courses where students put on lab coats and safety goggles as they become familiar with standard laboratory equipment and protocols and the technical knowledge of chemistry;

- a communication course where students visit schools as the subject matter expert to present a scientific or engineering concept to elementary or secondary students;

- a portfolio course where students reflect on their work in courses and build a master résumé;

- an environmental chemistry course and a plant design course where students operate as a team in a simulated consultancy project.

In each of these contexts students are on "the threshold between current reality and future possibilities"[6]. Students are certainly trying out roles and ways of being; they are also getting responses to these new ways of being from their peers, from instructors, and from the context participants. Although students are expected to commit to playing the role the stakes are arguably qualitatively different from those in a work context; commitment can be provisional. The purpose of the identity activity is also different. In identity work an individual works to 
claim an identity within an institution and to be granted that identity by the powers of the institution. In the activities the students engage in, they are exploring the different identities that may or may not lead to a desire to make a more definite commitment. Most often they are working to claim and be granted the identity of 'successful engineering student' - related to what Gibbs and Simpson, quoting Miller and Parlett (among others), describe as a focus on "cues about what was rewarded in the assessment system",[11]. As a result, students focus on what will get them through - preferably with good marks. This focus can sometimes work against learning (and the development of professional identity); as participant Simon pointed out,

we are absorbing a lot of [...] information very quickly. The difficulty I think we all have $[\ldots]$ is being bombarded by assignments and midterms on a very, very regular basis. [...] And it's the constant bombardment leaves us very little time for absorption of information. [...] I think in terms of our overall learning, the constant bombardment with assignments and midterms is actually detracting from our learning.

What marks the particular courses we looked at is an effort to slow down that process through building in iteration, reflection and multiple sites of interactive response.

There are several aspects of the identity process that inform our study. One aspect includes the prior experiences, beliefs, values and knowledge each student brings to the current site (the undergraduate engineering program). A second aspect comprises the experiences, beliefs, and values the current engineering program and the chemical engineering program, in particular, espouse explicitly and implicitly. A third aspect emerges from the interaction of the students with the current sense of engineering identity as they 'try on' or 'try out' different ways of being a chemical engineer in different settings. It is important to understand that the identity process includes both what an individual asserts about her or himself as well as what the context ascribes. There is often both a tension between the asserted and ascribed identity as well as a 'fit' between the two. Although much of the identity scholarship examines this tension and how individuals seek to manage it, that is not the focus of our research. Our focus remains more on the ways in which students explore and try on variations of a professional identity within the curriculum and student experience.

\section{A closer look at one participant: Téa}

Téa made her first professional public presentation in the first term of her first year as a chemical engineering student. She presented, as part of a team, an experiment meant to show the concept of bonding and the microscopic composition of matter to a small group of grade one students. In her personal reflection, a short narrative organized as a "what I did, what I did well, what I could have done better and what I learned," she singled out "learning to adapt our language based on the knowledge level of the audience." The language choices made in subsequent documents produced for her various courses provide evidence of how she applies this realization as part of her developing definition of "professional" and the identity she tries on as she makes these language choices.

${ }^{2}$ All quotations attributed to Téa are taken from interviews, discussion groups and written assignments from the 2012-2013 academic year. 
She also explicitly identifies characteristics of professional writing in a 'contextual statement' that accompanied one of the artifacts in her portfolio. In the contextual statement she concludes, "the skill of expressing written materials concisely and scientifically learned from this experience will be valuable in my future career." She enumerated organizing the document so that a reader "can easily and quickly locate information" with the "use of numbered sections, bolded and underlined section headings, differentiated use of bullet and numbered lists." She also identified the use of passive voice, past tense, use of visuals and proper referencing as hallmarks of being "professional and objective." These were affirmed by her communication instructor as being important to professional communication and that her work exemplified them. The following preliminary findings are based on an analysis of her work both individually and with her team as well as comments made during interviews and discussion groups.

We looked Téa's work from three key courses, CHE 230, CHE 297 and CHE 298. The secondyear environmental chemistry course (CHE230) consulting project emphasizes the sites of interaction between instructor/client and student/consultant, as well as between students on a single consulting team; as a result, it became a rich source of data on IR and identity activities. In the Communications Portfolio course (CHE297), students had to write contextual statements on two items from their course work (one of these items was often an assignment from CHE230). In the second year communication course (CHE 298) students were required to do both an individual and group reflection on their school visit ${ }^{3}$. The reflections in these statements-and the corresponding instructor comments - are an important tool in the identity work and play process $^{[7]}$ as well as a site of IR.

We used the initial analysis of the written interim proposal and the interim client meeting in CHE230 to begin to categorize the nature of Instructor IR, which, as it turns out, tends to have many nuances in terms of focus and delivery (see Table 1). Instructor IR focused on four main subject-areas: content-specific, dealing with the technical elements of the proposals; rhetorical strategies, dealing with the ways in which claims are articulated and substantiated; formatting, dealing with the elements of document design and layout; and professional language, dealing not only with elements of English language usage but also with conventions in professional engineering. The manner of delivery fell into a few clearly identifiable categories in each subject-area, in most cases taking the form of questions, directives, a noting of conventions or affirmations. The professional language subject-area had further unique characteristics of delivery, some around error (explanation, identification, correction) (see, for example, $\mathrm{Li}^{[15]}$ and Ellis $^{[20]}$ ) and some around conventions typical to engineering practice. We then returned to the specific instructor responses, categorized and quantified them. Simultaneously we examined the text in the interim proposal and the final proposal to determine what, if any, changes had occurred. What we found is discussed below.

${ }^{3}$ Students visited public schools in small teams as subject matter experts to present different scientific and/or engineering concepts to students. Teams were assigned to classes that ranged from grade one to high school. This was their first 'public' presentation as a chemical engineering student. 
Table 1: Categorizing Instructor Interactive Response

\begin{tabular}{|l|l|}
\hline Focus & Delivery \\
\hline Content-specific & Questions \\
& Directives \\
& Conventions \\
& Affirmation \\
\hline Rhetorical Strategies & Questions \\
& Directives \\
& Conventions \\
& Affirmation \\
\hline Formatting & Questions \\
& Directives \\
& Conventions \\
& Affirmations \\
\hline Professional Language & Error explanation \\
& Error identification \\
& Error correction \\
& Conventions \\
\hline
\end{tabular}

Examples of the application of both language choice and Téa's self-identified characteristics of professional communication are in evidence in her work with her team in CHE 230. The course is set up to simulate a consulting project. Students work in teams of five, select one of three contaminated sites (water, soil or air) and propose a strategy for dealing with the contaminants. Each team meets twice with 'representatives' (the course instructors) from a fictitious company that put out the request for proposals based on real-life scenarios. The first time is an interim proposal meeting and the second time is a more formal client meeting where the team presents their final proposal. In the first meeting the boundaries between classroom and simulation are blurred. The instructors ${ }^{4}$ will often break out of role to offer advice or comment on student performance as consulting environmental engineers. Sometimes the advice is technical while at other times it is directed at the professional performance. In the final client meeting where the team presents their proposal accompanied by a formal written proposal, the roles are more strictly adhered to until the meeting concludes, at which point the instructors step out of their 'client' roles to give immediate feedback on the presentation. In this course, students have the opportunity to 'try on' the roles, the language, the values and behaviors of a consulting engineer. For some students this provides the first glimpse of what a chemical engineer's work may include and as such develops the student's understanding of the profession and his or her own aspirations within the profession.

From the feedback on the interim proposal of Téa and her team (one of whom is also a participant in our study) and the changes made to the proposal it is possible to see how the team

${ }^{4}$ One instructor, Sylvie, is a lecturer with the Engineering Communication Program, the other, Scott, a sessional lecturer in Chemical Engineering, currently a graduate engineering student who has returned to study after several years as a successful consulting engineer. They operate as a team where the boundaries between areas of expertise are not always clearly drawn or adhered to. 
revised their document — language, presentation, content — and changed the character of it from a student report to a professional proposal. Some of the changes were simply physical changes in layout and spacing. The interim proposal had a table of contents but it was single spaced and crowded onto only three-quarters of the page. One instructor made the comment: "adding a few blank lines would make this much easier to read." This is an example of a comment categorized as having a focus on formatting and as directive. The team reformatted the table with blank lines between each entry that made it much easier to read and relieved the crowded, over-stuffed appearance. We often see students try to beat page limits by decreasing font sizes, margins and line spacing. We also see students afraid to use white space in layouts, a hallmark of more professional layouts. In this case, the margins were also changed throughout the document. The left and right margins were widened so that the text field was narrower making the pages appear less crowded, the lines of text shorter and easier to scan.

The bulk of the changes between the interim proposal and the final proposal were substantive content changes. In the project that Téa did with her team, the instructor's written comments on their written interim proposal focused mostly on the content-specific $(71 \%$ or 14 comments out of 17). While there were some questions (3), directives (1) and affirmations (1), the majority of the content-specific comments concerned conventions of practice and presentation in the discipline. It is expected that the students will not have all of the knowledge and information they need when they present their interim proposal, although they are expected to have done sufficient research to speak intelligently to standard practices. They are also expected to apply their knowledge of chemistry, environmental safety, sampling and laboratory techniques to a real-life problem of chemical contamination in the environment. In Téa's team's work the comments from Scott were taken seriously and addressed in major revisions within the document and additional research. The result can be characterized as a transformation of what was a mashup of student research report on what kinds of protocols, testing and analyses are done in air quality control studies and a proposal to a company to identify contaminants believed to be responsible for chronic cough reports around a particular location. The final report no longer displays knowledge students believe is being required. Instead, it lays out a specific set of protocols supported by reference to industry standards and practices. The document displays the application of knowledge in a simulated professional problem. The following excerpts from the interim and final proposals demonstrate this.

In a table in the interim report, Scott included a number of content questions and directives:

- "that's really low. Can you detect that low?"

- "asbestos?"

- "should also have a list of what sampling and analytical methods will be used for each and the laboratory reporting limit"

In the final report the table was completely reorganized and expanded. The team not only addressed the specific comments above, but also expanded the scope of the table to include sampling method, sampled gas or contaminant, health effects, concentrations limits, sampling frequency, analytical method and accepted detection limits.

Comments from the Sampling and Technical Analysis section resulted in a complete revision of this section as well. The comments, a combination of questions ("not really clear what this means. If you don't have soil gas samples, how do you know which compounds are potentially 
originating in the subsurface?") and content conventions ("Summa cans are not suitable for SVOCs, PAHs, PCBs, microbials, radon and particulates"), were addressed through additional research and the addition of new material that was more specific both in method and goals. It was presented with a different audience in mind, a knowledgeable client who would make a judgment on the quality of the content, the reliability and validity of the claims (including budget numbers) rather than just a display of knowledge.

It is possible to see Téa in the interim proposal meeting putting on the role of professional consulting engineer, specifically the team role she identified for herself. She sits up straight, pen in hand, eyes focused on the presenter and then the clients, making notes and nodding as she listens to their comments and questions. These are the notes she refers to in her personal reflection that she would bring to the attention of other team members in order to ensure that they incorporated or "fixed" the part of the documents they were responsible for before the proposal was finalized. Interestingly, some of this professional identity falls away when she becomes a "student from Shanghai" and hedges on being the expert (perhaps experiencing what Ibarra and Petriglieri ${ }^{[6]}$ categorize as the negotiation between multiple identities).

The two instructors' verbal discussion in the interim client meeting was more varied in focus than their written feedback, with $40 \%$ (10 out of 25$)$ of comments dealing with content, $36 \%$ ( 9 out of 25) dealing with rhetorical strategies and 24\% (6 out of 25) dealing with formatting. Here, too, however, a significant number of all comments were framed as engineering conventions $(40 \%$ or 10 out of 25$)$.

The centrality of engineering conventions is perhaps not surprising, given that the project is a simulation of a consulting project and that the instructors also double as clients. The instructors' goal - both in the nature of the assignment and in the framing of their responses - seems to be pushing the students toward the professional practice of engineering. As Scott pointed out to the students during the interim client meeting:

And so through these interactions along the way, we're gonna keep getting you closer and closer to what we expect but we're not just gonna give it to you and say just do this and you're fine. 'Cause that makes it all a little too easy. [...] As you read through our comments, we might make a comment on one thing in one location, but ask yourself, does this comment apply in other locations? Because if we have the same comment over and over again, we're not going to type it twenty times. So it's up to you [to] take what we have to say and say, okay, they're steering me in this direction, I'm going to take it even further in that direction.

Central, too, to the meeting is the presence of affirmation on all areas $(28 \%$ as opposed to only $12 \%$ in the written comments). Repeatedly throughout the meeting, the instructors highlight elements of the proposal - content, rhetorical strategies, formatting and, indirectly, professional language — that the team did well.

The discussion in the interim client meeting is meant to complement the written comments on the interim proposal, with the latter providing more details on the high-level commentary in the former. But it is also an opportunity for the "feedback" to be truly interactive. Students have a 
chance to ask questions - and here the dual roles of instructor/client and student/consultant come into sharp relief:

[Student]: Our next question was about the budget. I was wondering about the budget, the ideal budget for this project, 'cause maybe overall or ...

Scott: Yeah, well, you see that's a kind of question unfortunately as a consultant you never ask a client in a competitive bid situation. Because if I tell you how much, you know, I have to spend, you might be saying, I can solve this problem for $\$ 300,000$, and I say I've got a half a million dollars to spend and all of a sudden you think, wow, we've got $\$ 200,000$-worth of profit. And my boss would kill me if I told you that I have that much money, you know what I mean? So, realistically, though, I have provided hints for groups that are really missing the mark in terms of how much in total, and I've given you comments as well in your budget that sort of steer you in a general direction. But I'm not giving you an amount.

The student/consultant's question about an "ideal budget" is not directly answered - clearly in keeping with the strategy of having the students reach these conclusions on their own. However, the response to the question also shows the fluidity between the instructor and client role - and how a client would typically behave in this sort of situation. The "instructor" will not provide an answer for pedagogical reasons; the "client" will not provide an answer because, from a professional standpoint, an answer would be bad business practice.

Both instructors and students can also respond to or elaborate upon each other's comments. Consider the following exchange around inclusion of recommendations in a table in the proposal:

Sylvie: As Scott pointed out, you've used tables quite effectively to lay out information in a really succinct way. You have a column that's recommendations for improvement, and I wondered if [Sylvie looks at Scott] —I don't know how you feel about this-but I wondered if you take this further and, pushing against the word limit, whether that could be contained elsewhere, whether that might be presented in the appendix as opposed to the body of the document.

Scott: Yeah, good point. If you think about where this all ends up, ultimately I want somebody to tell me what's my problem so that then I can move forward with fixing it. [...] As soon as you write something in your report that says we recommend this, then I've got this document that lives forever that says your consultant recommended that you do that. If I don't do it, somebody's going to ask the question, Why didn't you do what your consultant recommended? So most clients don't actually want you to write recommendations until you've actually had a chance to talk and really figure out that these are the things we absolutely are going to do moving forward.

Picking up on Scott's earlier affirmation, Sylvie begins to question the inclusion of recommendations in the particular table. Her question-both to Scott and, indirectly, to the students - begins by dealing with a formatting issue (moving something from the body of the document to an appendix). Scott agrees initially ("good point"), but he immediately moves the discussion in the direction of content conventions, pointing out that recommendations at this stage in the proposal can be problematic for a client, setting up a potential liability. The interaction between the two instructors is partly based on their different backgrounds-Sylvie a 
communication instructor and Scott an instructor who is also a consulting engineer. It is interesting to note, however, that both are blurring the instructor/client boundaries; even Sylvie's initial question may stem from a client's desire for a more succinct document.

Students in CHE230 were also required to write two reflections on their process in executing the consulting project. Here, we were able to identify some sites of peer IR (although these were accounts, rather than observations of the actual sites). The first reflection-written by Téa and her team-provides little in the way of specific descriptions of interactive response, although it does include reference to affirmation in the delivery of peer feedback: "team members have been quite patient and encouraging of all possible ideas that may help enhance the overall quality of all deliverables" and "we also hope to continue fostering a positive and encouraging environment." The second reflection, however (written by Téa individually), provides more detail in the delivery of peer feedback - likely to do with content, but also possibly with rhetorical strategies and formatting (she refers to feedback given by the "clients" in the interim meeting). Here, peer IR takes the form of questions - "I always read over others' work and ask questions when I don't understand" - and directives - "I took notes during the first client meeting and told teammates to fix their parts when editing our proposal." Directives also occasionally take the form of taking responsibility for a particular task; as Téa points out, "I always format our work when everyone is done."

Téa chose to reflect on the formal client meeting (and corresponding handout) from CHE230 in her CHE297 portfolio. In her contextual statement, she reiterates the manner in which the team engaged in peer IR: "while rehearsing with each other, we were able to express our ideas freely, listen to others' comments attentively and reach a consensus together." But she goes further in demonstrating an internalization of her instructors' feedback, using their directives on formatting from the interim proposal in creating the final meeting handout:

Our meeting handout served as a visual aid with limited words because we wanted to sustain the professionals' attention on our presentation, instead of making them distracted with our texts. The pages, figures and tables were clearly labeled with numbers to help us refer to materials easily.

The instructors' criteria for what constitutes a professional document have now become the student's criteria as well.

The instructor IR on the contextual statement - given by Len, another communication instructor - has a very different focus than in the CHE230 assignments. Here, the majority of Len's comments - $57 \%$ or 8 out of 14 -involved error correction of professional language, all instances of rephrasing (for example, "conduct effective teamwork" becomes "work effectively in a team"). The remaining comments all focus on affirmation: 7\% (1 each) dealing with content and formatting, and 29\% (4 out of 14) dealing with rhetorical strategies. There may be several explanations for this difference in instructor IR focus. First, the nature of the assignment is reflective rather than technical; as a result, the focus of the instructor's comments may be directed more toward affirming the student's own assessment of her work. Second, while Téa did include the actual item - in this case, the handout and script from her final client meeting - Len is not as familiar with that assignment, and as such may not feel comfortable contradicting Téa's assessment. And finally, perhaps because of that lack of familiarity, Len resorts to feedback on elements that he is more confident about — namely, language error correction. Having said that, it 
is important to point out that Téa exceeded three out of the four requirements on the rubric (structure and organization, background and context and persuasiveness) and met requirements on completeness; the absence of corrective feedback on content, rhetorical strategies and formatting likely point to her success in these areas.

\section{Conclusions/Moving Forward}

The findings in this paper represent the analysis and interpretation of a small part of the data we have collected to date, focusing on only two sites of learning-identity and interactive response - and only one of our participants in the first year of the study. Having said that, even here, we have been able to determine clear links between the conceptualization and evolution of identity and interactive response. We have also been able to see an evolution in Téa's professional acts and her conception of what it means to be a professional engineer. Through the interplay between the various engineering cultures and practices she participates in, she begins to identify key elements of a professional self: the need for tailoring language to particular audiences, being concise, organizing materials effectively, referencing and using visuals. And, she begins to put these elements into practice as she moves from context to context. For example, she comments that her use of graphics in her first lab report stemmed from her need to visualize what was being described, but she expands that initial very personal need as she sees the value of visuals in a document and a presentation as she indicates here-particularly in the reflection on her CHE230 final client meeting handout (transferring what she was told in the CHE230 interim client meeting into her future work). This continued emphasis on effective visuals is also evident in the work we are seeing from her this year (e.g., a written lab report produced for CHE324, the Chemical Process Design Laboratory). We will continue to analyze our data with these kinds of trajectories and links in mind for all of our participants.

As with most large research projects, these preliminary findings open up a great many more questions than they answer. How do different agents/contexts affect the student's sense of identity over time (and are interactions with some agents or in some contexts privileged over others)? How does what we observe in this one participant's experience compare with the other participants? As students move out into the field of work - through internships, for examplewhat are the sites of interactive response, how are they different than what is experienced in the school environment and how do they influence the development of professional identity?

Our findings will also ultimately be influenced by the context in which we are working. The inclusion of research assistants this year (and in coming years) invariably informs how we shape the project. Input from our colleagues - particularly a group that meets regularly about engineering education research - may inform directions we take. And, perhaps most importantly, the continued input from our participants on our findings - which we share at regular intervalswill influence how we make connections between sites of identity and interactive response (indeed all the sites we have identified). The dynamic nature of the process-like identity itself-will continue to inform how we search and what we find. 


\section{Bibliography}

1. Tonso, K.L., Student engineers and engineer identity: Campus engineer identities as figured world. Cultural Studies of Science Education, 2006. 1(2): p. 273-307.

2. Brickhouse, N.W., M.A. Eisenhart, and K.L. Tonso, Forum identity politics in science and science education: Identity politics, learning, and academic success. Cultural Studies of Science Education, 2006. 1(2): p. 309-324.

3. Walker, M., Engineering identities. British Journal of Sociology of Education, 2001. 22(1): p. 74-89.

4. Markus,H. and P. Nurius, Possible selves. American Psychologist, 1986. 41(9): p. 954-969.

5. Ibarra, H., Provisional selves: Experimenting with image and identity in professional adaptation. Administrative Science Quarterly, 1999. 44(4): p. 764-791.

6. Ibarra, H. and J.L. Petriglieri, Identity work and play. Journal of Organizational Change Management, 2010. 23(1): p. 10-25.

7. Eliot, M. and J. Turns, Constructing professional portfolios: Sense-making and professional identity development for engineering undergraduates. Journal of Engineering Education, 2011. 100(4): p. 630-654.

8. Hattie, J. and H. Timperley, The power of feedback. Review of Educational Research, 2007. 77(1): p. 81-112. 9. Brown, S., Assessment for learning. Learning and Teaching in Higher Education, 2004-5. 1: p. 81-89.

10. Hattie, J., Influences on student learning. Inaugural Lecture: Professor of Education, University of Auckland, 1999.

11. Gibbs, G. and C. Simpson, Conditions under which assessment supports student learning. Learning and Teaching in Higher Education, 2004-5. 1: 3-31.

12. Wood, D., J. Bruner and G. Ross, The role of tutoring in problem solving. Journal of Child Psychology and Psychiatry, 1976. 17: p. 89-100.

13. Sugita, Y., The impact of teachers' comment types on students' revision. ELT Journal, 2006. 60(1): p. 34-41.

14. Niven, P. and B. Meyer, understanding the impact that principled formative feedback has on first year students writing: Is it useable or not?: An action research project. International Journal of Learning, 2007. 14(8): p. 13-22.

15.. Li, S., The Effectiveness of Corrective Feedback in SLA: A Meta-Analysis. Language Learning, 2010. 60(2): p. 309-365.

16. Poehner, M.E., Dynamic assessment as a dialectical framework for classroom activity: Evidence from second language (L2) learners. Journal of Cognitive Education \& Psychology, 2009. 8(3): p. 252-268.

17. Poehner, M.E., The zone of proximal development and the genesis of self-assessment. The Modern Language Journal, 2012. 96(4): p. 610-622.

18. Kozulin, A. and E. Garb, Dyanic assessment of literacy: English as a third language. European Journal of Psychology of Education Special Issue: Vygotsky's Theory in the Classroom, 2004. 19(1): p. 65-77.

19. Anghileri, J., Scaffolding practices that enhance mathematics learning. Journal of Mathematics Teacher Education, 2006. 9(1): p. 33-52.

20. Ellis, R., A typology of written corrective feedback types. ELT Journal, 2009. 63(2): p. 97-107. 


\section{Appendix A: Details on Data Collection and Methodology}

\section{Data Collection}

Eleven (11) students from the 2012-2013 CHE second-year class elected to participate in the study. We will follow these students through to their graduation and one year afterward.

Ten (10) faculty (including research- and teaching-stream faculty, sessional lecturers and teaching assistants) from across three core second-year courses elected to participate in the study in 2012-2013; in 2013-2014, nine (9) faculty (including teaching-stream faculty and teaching assistants) from across two core third-year courses elected to participate in the study. Courses were chosen based on our previous research, notably those that garnered the most frequent mentions from former students as to the impact on their professional development. These included:

- CHE297, Communications Portfolio I

- CHE298, Communication

- CHE230, Environmental Chemistry

- CHE324, Chemical Process Design Laboratory

- CHE326, Thermodynamics and Kinetics Laboratory

Individual interviews were conducted with all participants. Students were interviewed twice (once at the beginning and once at the end of the year); faculty were interviewed once (at the beginning of their course). Interviews were video recorded and transcripts created.

Discussion groups were held with all participants. We met with students twice (once at the end of each term). Faculty discussion groups occurred once each term: one-term courses (CHE298, CHE230, CHE324, CHE326) had one meeting; year-long courses (CHE297) met twice. Discussion groups were video recorded and transcripts created.

In all cases - interviews and discussion groups - participants were provided with a list of questions/topics in advance (see Table A1). Topics emerged out of our initial research questions on the nature of engineering identities and how/where they emerge/evolve.

In the initial interviews, we were concerned with establishing a baseline of definitions/ perceptions of key areas, including "professionalism" and "communication." We also focused on collecting narratives of particular experiences from the participants that highlighted their points of view. We used subsequent interviews to revisit key issues raised earlier; in the first year of the study, students were provided with a copy of their first interview (video and transcript) prior to the second interview.

With the discussion groups, we explored further some of the key issues identified by participants in their interviews. In addition to further discussion on "professionalism" and "communication," notions of mastery and expectations - and instances of conflict here - were addressed. 
Table A1: Interview and Discussion Group topics

\begin{tabular}{|c|c|c|}
\hline & Individual Interviews & $\begin{array}{l}\text { Discussion Groups } \\
\end{array}$ \\
\hline $\begin{array}{l}\text { Student } \\
\text { Participants }\end{array}$ & $\begin{array}{l}\text { - Educational background } \\
\text { - Linguistic history } \\
\text { - Meaningful course or extra- } \\
\text { curricular experience(s) }\end{array}$ & $\begin{array}{l}\text { - } \text { Mastering course content } \\
\text { - Trade-offs required in } \\
\text { academic career } \\
\text { - } \text { Coping with stress } \\
\text { Professionalism in } \\
\text { - } \text { Beingineering a chemical engineer }\end{array}$ \\
\hline $\begin{array}{l}\text { Faculty } \\
\text { Participants }\end{array}$ & $\begin{array}{l}\text { Academic and industry } \\
\text { experience } \\
\text { Experiences/activities in } \\
\text { course that contribute } \\
\text { to/promote student } \\
\text { understanding of } \\
\text { professionalism and/or } \\
\text { communication in engineering }\end{array}$ & $\begin{array}{l}\text { Defining professionalism } \\
\text { - } \\
\text { Defining professional } \\
\text { communication } \\
\text { Examples of activities/student } \\
\text { performance of } \\
\text { professionalism and } \\
\text { professional communication } \\
\text { - Expectations vs. student } \\
\text { performance }\end{array}$ \\
\hline
\end{tabular}

We collected a number of assignments from each of the courses (see Table A2). Faculty provided course syllabi, assignment instructions and rubrics. When possible, we collected iterations of student deliverables (e.g., the Master Résumé in CHE297). Written deliverablesincluding feedback from instructors - were collected electronically, in hard copy or - in the case of verbal feedback - by video recording. Oral deliverables - including feedback from instructors when given - were video recorded, when possible in situ (CHE230, CHE326), occasionally in a separate time slot outside of class (CHE298).

We collected deliverables with significant communication components (written, oral, visual), done both individually and in teams. Our selections were also made based on acquiring a range of genres (e.g., résumés, proposals) and purposes (e.g., informative, persuasive, reflective).

Three of the 11 student participants self-identified as second language learners. We asked each of these three students to keep a language log for one month of each term where they documented their language use in school work.

One of the investigators (Deborah Tihanyi) took on the role of participant/observer in the second-year lab course,

- participating in weekly lab work, including setting up and running experiments and working with partners when assigned;

- auditing quizzes and term tests;

- keeping an ongoing journal documenting her experiences each week;

- maintaining a Facebook page — inviting students to participate - documenting key events/accomplishments in lab and other related issues and 
- debriefing monthly with the co-investigator on key issues pertaining to the study.

Table A2: Course work collected from students. Note that instructor feedback was also collected for each deliverable, including rubrics, summative comments and/or video recordings of oral feedback. Work is individual unless otherwise noted.

\begin{tabular}{|c|c|c|}
\hline & Written Deliverables & Oral Deliverables \\
\hline CHE297 & $\begin{array}{ll}\text { - } & \text { Master Résumé (multiple } \\
& \text { iterations) } \\
\text { - } & \text { ePortfolio } \\
\text { - } & \text { Contextual Statements }\end{array}$ & \\
\hline CHE298 & - Reflection on School Visit & $\begin{array}{l}\text { - Oral Presentation with hand- } \\
\text { drawn slide } \\
\text { - Oral Presentation using } \\
\text { PowerPoint }\end{array}$ \\
\hline CHE230 & $\begin{array}{ll}\text { - } & \text { Interim Proposal (team) } \\
\text { - } & \text { Final Proposal (team) } \\
\text { - } & \text { Reflections on teamwork and } \\
& \text { leadership style } \\
\end{array}$ & $\begin{array}{ll} & \text { Interim Client Meeting (team) } \\
\text { - } & \text { Final Client Meeting (team) }\end{array}$ \\
\hline CHE324 & - Written Lab Reports & \\
\hline CHE326 & & $\begin{array}{l}\text { Oral Lab Reports (six in total } \\
\text { for the course) }\end{array}$ \\
\hline
\end{tabular}

\section{Methodology}

Our main method of analysis is the continuous review of data. We began with a list of thematic categories we had identified in our previous research — review of past portfolios and focus groups - including community, identity, practices, feedback and paradoxes. With this list, we went through the transcripts and video recordings of interviews and discussion groups; with multiple passes through the data - both on our own and together-we were able to expand and refine these thematic categories.

We are making use of NVivo as our primary tool of data analysis. All video recordings, transcripts, documents and social media text are fed into NVivo, which allows us to code data based on common thematic criteria. NVivo analysis allows for quantitative and qualitative comparison across data sets. 